\title{
RETRACTION
}

\section{RGD-FasL Induces Apoptosis in Hepatocellular Carcinoma}

\section{Zhongchen Liu, Juan Wang, Ping Yin, Jinhua Qiu, Ruizhen Liu, Wenzhu Li, Xin Fan, Xiaofeng Cheng, Caixia Chen, Jiakai Zhang and Guohong Zhuang}

Cellular \& Molecular Immunology (2013) 10, 183; doi:10.1038/cmi.2012.75

Retraction to: Cellular \& Molecular Immunology 2009; Volume 6, Number 4, page 285-293. doi:10.1038/cmi.2009.38.

The corresponding author has indicated to the journal that this paper should be retracted as it is a duplicate publication. The author apologizes to the journal and its readers for any inconvenience caused. 